\title{
Managing bowel and bladder impairments in sacral chordoma patients: a case-based approach
}

\author{
Sasha E. Knowlton ${ }^{1} \cdot$ Cody Andrews $^{1} \cdot$ Corey Bindler $^{1} \cdot$ Lisa M. Ruppert $\mathbb{D}^{1,2}$
}

Received: 14 April 2017 / Revised: 15 September 2017 / Accepted: 21 September 2017

(C) International Spinal Cord Society 2017

\begin{abstract}
Introduction Chordomas are primary bone tumors that occur in the axial spine and most commonly in the sacrum. Because of their location, chordomas can affect bowel and bladder continence resulting in either an upper or a lower motor neuron functional pattern.
\end{abstract}

Case presentation We present two cases describing chordoma's impact on bowel and bladder function and the management plan used for improvement.

Discussion The accurate identification of an upper or lower motor neuron pattern in the setting of chordoma aims to significantly improve management of bowel and bladder impairment.

\section{Introduction}

Chordomas are a type of primary bone tumor that occur in the vertebral bodies. Chordomas account for $17.5 \%$ of all primary malignant bone tumors within the axial spine [1]. The incidence of chordoma was 0.08 per 100,000 with an average age of diagnosis at 58.5 years in a Surveillance, Epidemiology, and End Results survey [2]. In this population, the 5-year survival rate was $67.6 \%$ and the 10-year survival rate was $39.9 \%$ with a median survival time of 6.29 years [2]. Chordomas are slow-growing and can recur but have a low rate of metastases [3-5]. Almost half of all chordomas occur in the sacrum [6-11].

Comprehensive rehabilitation of chordoma patients is important to improve functional independence and quality of life as these patients have multiple impairments. Chordomas can result in myelopathic sensory changes, weakness, bowel, bladder, and sexual dysfunction, depending on tumor location and nerve involvement [1].

Sasha E. Knowlton

sasha.knowlton@gmail.com

1 Rehabilitation Medicine Service, Department of Neurology, Memorial Sloan-Kettering Cancer Center, New York, NY, USA

2 Department of Rehabilitation Medicine, Weill Cornell Medical College, New York, NY, USA
In terms of bowel and bladder function, the preservation of the bilateral S3 nerves is extremely important [7, 12-14]. There has been limited research regarding bowel and bladder dysfunction in the chordoma population. Identification of either an upper motor neuron (UMN) or lower motor neuron (LMN) bladder, and bowel pattern in the setting of chordoma is extremely important in order to provide ideal management and continence for quality of life and independent functioning. This article presents two cases of chordoma with LMN impairments in bladder and bowel management.

\section{Case presentation}

\section{Case 1}

A 25-year-old female developed lower back pain in the setting of a mass at S3-S4 seen on MRI (Figs. 1a and b) with later biopsy confirming sacral chordoma. Repeat MRI 3 months after diagnosis demonstrated tumor progression and CT scan demonstrated new lung nodules concerning for metastases 4 months after diagnosis. Despite starting a targeted chemotherapy (EZH2 inhibitor), she had tumor progression. She underwent radiation to the left sacrum in 26 fractions for a total dose of $5200 \mathrm{cGy}$, followed by nine additional fractions totaling $1800 \mathrm{cGy}$. 

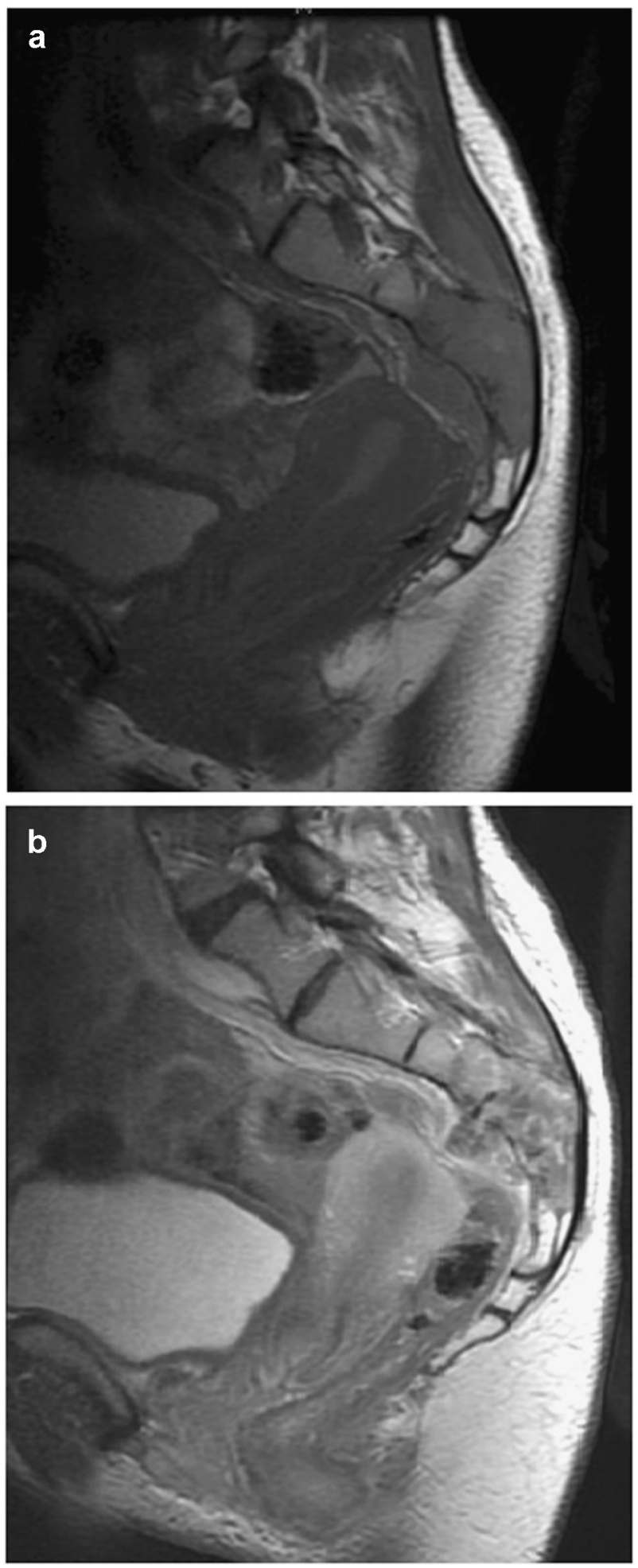

Fig. 1 MRI of Patient 1 at time of chordoma diagnosis. a demonstrates a sagittal T1-weighted image of a sacral chordoma for the patient in Case 1. b demonstrates a sagittal post-contrast T1-weighted image of the sacral chordoma at the same time point
She initially presented to physiatry for evaluation of urinary retention and constipation 5 months after diagnosis. Her symptoms of urinary retention and incomplete bladder emptying began prior to diagnosis, but worsened after she underwent ovarian transposition surgery, at which time she could only urinate when she had bowel movements. At presentation, she reported difficulty with urinary initiation, small volume voids and increased urinary frequency of every $30 \mathrm{~min}$ during the day. Manual pressure to the abdomen did not assist with emptying. She had tried multiple medications for bowel constipation including docusate, milk of magnesia and bisacodyl suppositories with poor success. She noted that she was able to move her bowels on polyethelene glycol, but that this medication often resulted in multiple bowel movements per day. Of note, she reported numbness around the rectum and sacral pain when toileting. She declined discussions regarding her sexual function at her initial and follow-up visits because of concerns about pain and her required oncologic treatments.

On examination, she had a full strength of her lower extremities but a palpable distended bladder and decreased light touch and pinprick sensation in the bilateral L5 and S1 dermatomes. Rectal examination demonstrated decreased sensation in the right S3-S5 dermatomes with intact sensation in the corresponding left dermatomes. She had intact deep anal pressure but delayed anal sphincter relaxation and weakened but present voluntary anal contraction. Bulbocavernosus reflex was not performed.

Based on her clinical presentation, her symptoms were attributed to a LMN pattern of injury of the bladder and bowel as the result of an incomplete non-traumatic spinal cord injury (SCI) from her chordoma with a L4 neurologic level of injury. She refused intermittent catheterization (IC) after receiving both teaching and a trial in clinic. As a result, a bladder program including bethanechol $15 \mathrm{mg}$ three times a day, leg elevation on a step stool and manual pressure through Crede's method was initiated. For her bowel program, her polyethylene glycol was continued and it was thought that the initiation of bethanechol could further promote emptying. She was recommended to start using a step stool when emptying to promote relaxation of the pelvic musculature. She refused manual removal of stool despite receiving teaching on technique and discussion of benefit.

Pelvic floor therapy was also initiated. Therapy initially focused on the external pelvic floor with gentle manual therapy and self-stretches to maintain mobility, improve sitting tolerance, and reduce myofascial restrictions from surgical intervention and radiation therapy, which were felt to be contributing to her impaired bowel and bladder function. External electromyography biofeedback was used to help retrain and coordinate pelvic floor muscles. Pelvic 
floor therapy also incorporated non-resistive strengthening exercises to increase hip and core stability.

At 2-week and 1-month follow-ups, her bladder program resulted in self-reported increased urinary volume, easier initiation of urine, improved stream without straining and less frequency. She had no incontinence episodes between voids on her program. Prior to initiating bethanechol, voiding volume was measured at $200 \mathrm{cc}$, and post-void residual demonstrated $148 \mathrm{cc}$ of retained urine. At followup, voiding volume was again measured at $200 \mathrm{cc}$ with
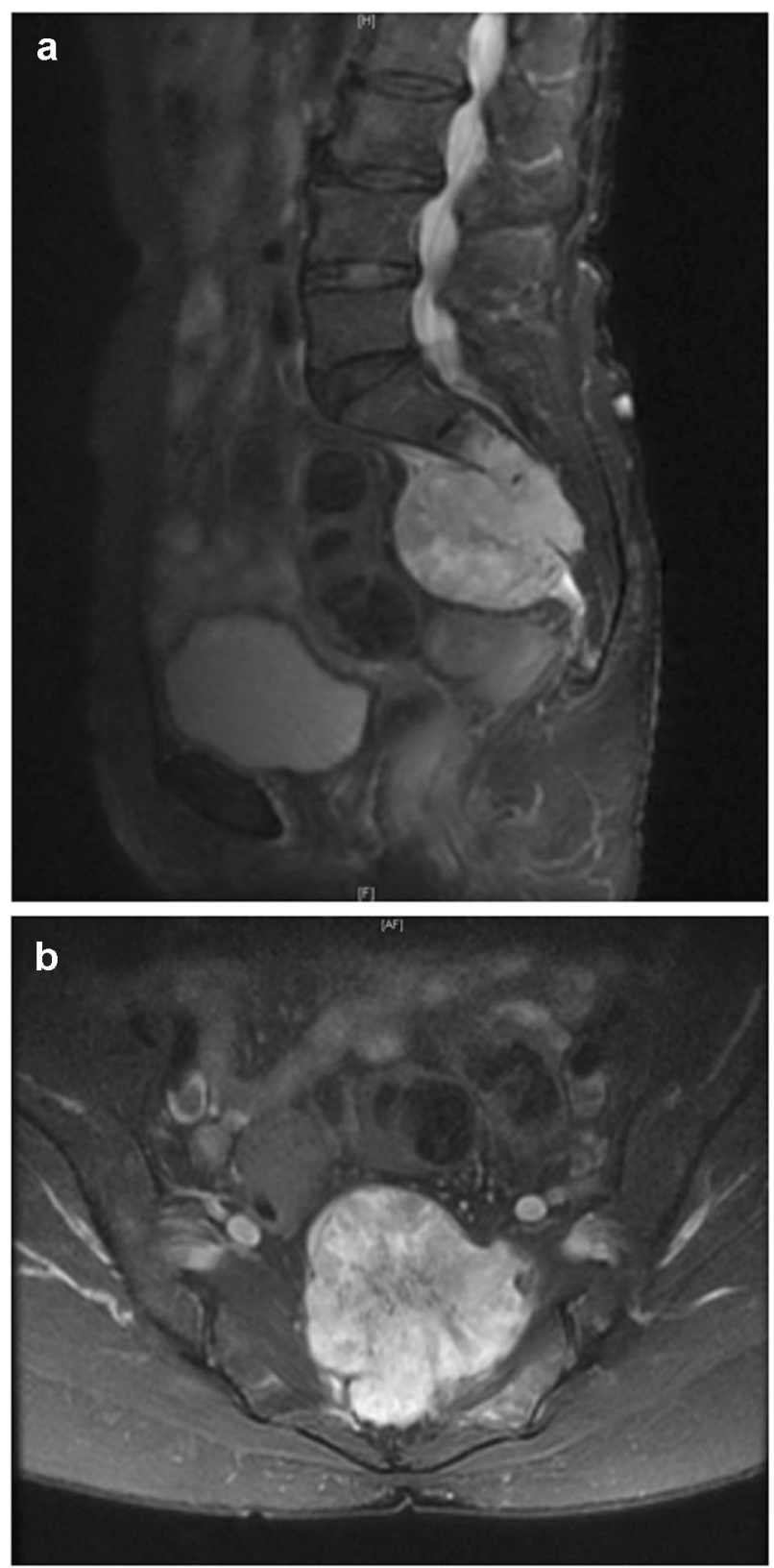

Fig. 2 MRI of Patient 2 at time of chordoma diagnosis. a demonstrates a sagittal T2-weighted image of the sacral chordoma for the patient in Case 2 at time of diagnosis. b demonstrates an axial T2-weighted image of the sacral chordoma at the same time point negligible measurement of post-void residual, suggesting complete emptying. Upper urinary tract anatomy was monitored during her treatment course via CT scans performed for cancer staging and showed no anatomic abnormalities; labs including BUN/creatinine were also completed and remained within normal limits. Urodynamic studies were considered however deferred due to improvements with initiation of her bladder program and difficulty with coordination during her cancer treatments. During her follow-up visits, she began having two successful bowel movements daily without incontinence. No abnormalities of the colon or rectum were noted on available imaging.

\section{Case 2}

An elderly female presented with left pelvic pain, vaginal numbness, urinary retention, and constipation. An MRI completed to evaluate her symptoms revealed a large sacral lesion extending to the S1-S2 junction with involvement of the left S2 and bilateral S3 nerve roots (Figs. 2a and b). Subsequent biopsy confirmed the diagnosis of chordoma. Radiation and resection were offered, but the patient elected to proceed with only proton beam radiation. She underwent a total of $7740 \mathrm{cGy}$ in 43 fractions. Her post-radiation course was complicated by low back and bilateral lower extremity pain attributed to lumbosacral radiculopathy and sacral stress fractures. Upon completion of her proton beam radiation, her bowel and bladder dysfunction improved significantly and her pain gradually resolved.

She presented to physiatry 6 years after her original diagnosis at the age of 84 with a 6-month history of worsening bowel and bladder incontinence. She reported that she began self-managing her constipation with polyethylene glycol and did attempt to manually disimpact herself, but had difficulty due to the softness of her stool. She also tried tap water enemas with limited success. She was started on IC three times daily by a urologist for urinary retention and overflow incontinence, but self-discontinued. She previously had urodynamic studies done at an outside institution, but records were unavailable at the time of physiatry evaluation. She reported she was informed the result showed she had a "weak bladder". She was instructed to submit the urodynamic studies for review.

MRI performed to evaluate her worsening incontinence revealed interval increase in size of the sacral chordoma and a distended bladder but no renal abnormalities (Figs. 3a and b). During physiatric evaluation, she reported stool incontinence with pasty consistency and near constant urinary leakage. She also reported progressive loss of sacral sensation, noting that she had no feeling of bladder or rectal fullness and was unable to feel when she was disimpacting herself or using an enema. Her symptoms had progressed to the point that diverting colostomy was recommended. 

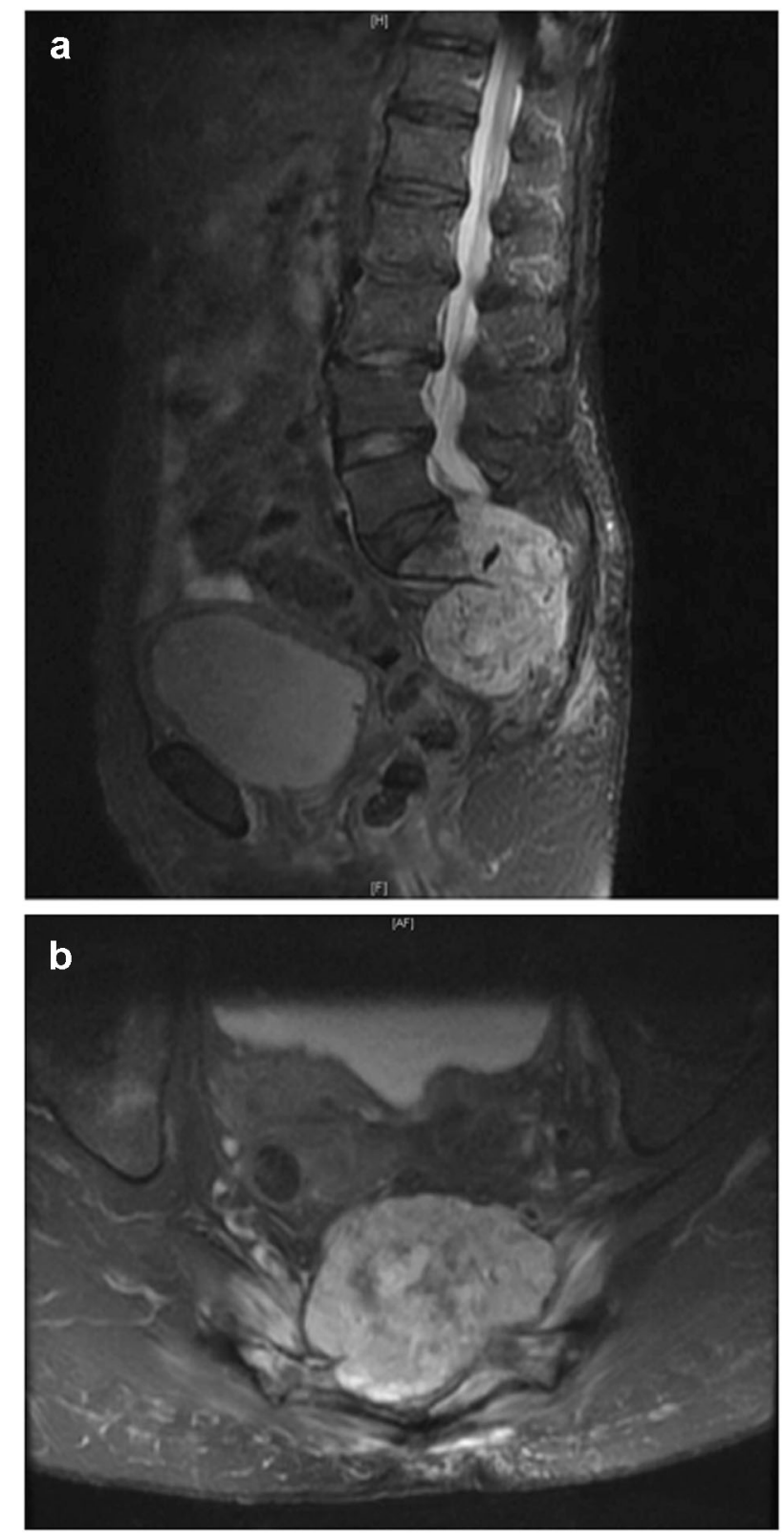

Fig. 3 MRI of Patient 2 six years after treatment with proton beam radiation. a demonstrates a sagittal T2-weighted image of the sacral chordoma for the patient in Case 21 -month prior to physiatry presentation and $\mathbf{b}$ demonstrates an axial $\mathrm{T} 2$-weighted image of the sacral chordoma at the same time point

Sexual function was not discussed as she did not feel it was important to her.

Physical examination revealed a mild decrease in left ankle dorsiflexion strength and impaired sensation in the bilateral S1 and S2 dermatomes. Her rectal examination demonstrated decreased S3-S5 sensation bilaterally with mild awareness to deep anal pressure and present but weak voluntary anal contraction. Bulbocavernosus reflex was not performed. Based on her clinical presentation, she was determined to have an incomplete non-traumatic SCI from chordoma with an L5 neurologic level of injury.

She was diagnosed with LMN dysfunction of bowel and bladder. For bowel management, she was recommended to continue polyethylene glycol and to add fiber supplementation for stool bulking, with both medications dosed together $\sim 8 \mathrm{~h}$ prior to an intended bowel movement. She was educated on proper technique for manual removal of stool and instructed to perform this intervention one to two times per day. For bladder management, she was recommended to return to self-IC every $4-6 \mathrm{~h}$ with goal of volumes less than $300 \mathrm{cc}$. Pelvic floor therapy was also initiated. During therapy, she was taught to optimize IC and manual removal to reduce pelvic floor strain and injury. Abdominal scar tissue management was initiated to aid evacuation and electromyography biofeedback was used to isolate and recruit the pelvic floor muscles to further promote continence.

At her 1-month physiatry follow-up, she reported good response to this treatment regimen. Her stools became bulkier and easier to remove, and she has been able to maintain bladder volumes of less than $250 \mathrm{cc}$ on her current catheterization schedule. She is no longer considering colostomy placement. She reported no incontinence episodes to bladder or bowel.

\section{Discussion}

The increasing survival rates of cancer patients have resulted in functional impairments from cancer and respective treatments. Chordoma patients in particular may develop non-traumatic SCIs as a result of cord compression with a variety of impairments based upon the spinal level of the tumor. These malignant SCIs differ from other SCIs as they are often incomplete injuries and patients have different life expectancies.

The majority of research thus far into functional outcomes in chordoma patients regarding mobility, bowel and bladder control has been conducted in the surgical literature [1, 6, 7, 12-24]. The importance of bowel and bladder control cannot be overstated for functional independence and quality of life. In the chordoma population, the pattern of either UMN or LMN bladder or bowels is important to identify based upon the tumor level and resultant level of resection. The pattern of injury is diagnosed based upon known level of tumor involvement and by rectal examination. Anal wink and bulbocavernosus reflexes should be performed to confirm diagnosis. Based on imaging and clinical findings, both cases had incomplete non-traumatic lower lumbar spinal cord injuries with LMN patterns of bowel and bladder involvement. Utilizing the International Standards for Autonomic Function, the patient in Case 1 
would have sacral function classified as reduced lower urinary tract awareness and continence while she had normal bowel sensation, but reduced continence and altered voluntary sphincter contraction [25]. The patient in Case 2 would be classified as absent urinary tract awareness and continence and absent bowel awareness and continence with altered sphincter contraction [25]. Given their routine cancer surveillance with laboratory studies and imaging that included the genitourinary and renal systems, we were able to monitor the effects of medication and other management strategies closely.

In the surgical literature, the preservation of bilateral S3 nerves after chordoma resection is noted to be important to maintain bladder continence [7, 13, 14]. Patients with bladder dysfunction should be placed on a bladder program to ensure emptying, most commonly with either a Foley catheter or an IC program [12, 16, 18]. Anticholinergic medications such as oxybutynin may benefit chordoma patients with UMN bladder dysfunction. Bethanechol or other cholinergic medications may assist LMN bladder emptying [26, 27]. LMN bladders can also utilize assisted emptying with manual pressure such as Credé. However, patients should not use Valsava maneuvers to void as it impacts emptying by increasing pressure against a closed sphincter. Patients may benefit from pelvic floor therapy to coordinate their muscles and reinforce appropriate behavioral strategies.

Both patients presented had LMN patterns of injury but varying levels of sacral sparing. Bladder programs initiated were based on clinical findings and patient preference, and both improved with interventions. A Foley catheter was not used in either case out of concern for recurrent infection, urethral trauma, impaired skin integrity, and the long-term risk of bladder cancer [28]. Post-treatment effects of radiation on the bowel and bladder if included in the radiation field could have possibly contributed to dysfunction in both cases, but this contribution was felt less likely, given examination and imaging findings.

Identification of a LMN or UMN bowel is also paramount for appropriate management. After sacral en bloc resection, those with intact bilateral S3 nerves had normal bowel function but resection of bilateral S2 or S3 nerves resulted in bowel dysfunction in all patients [7, 12-14]. Patients should have a goal of a daily or every other day movement with their bowel program. All chordoma patients should receive daily adequate hydration regardless of bowel pattern. Medications should be reviewed to screen for constipating agents. UMN bowel programs aim for a soft, formed, easy to pass stool with the use of softeners, irritants or suppositories. LMN bowel programs promote stool bulk and emptying pressure with fiber and laxatives; cholinergic medications such as bethanechol increase peristalsis and can also be considered in the management plan. Stool softeners should be avoided because these agents reduce pressure and do not assist with continence. Digital stimulation for UMN patterns and manual removal for LMN patterns may also be helpful for stool evacuation. Patients may benefit from pelvic floor therapy to work on scar tissue mobility and manual therapy in conjunction with electromyography biofeedback for pelvic floor muscle retraining. Both UMN and LMN patterns should not undergo large volume enemas because they can result in sphincter overstretching and increased rectal compliance.

In the case presentations, both patients declined to discuss sexual function for different reasons. However, in the setting of either UMN or LMN impairment, sexual function will likely be impacted [20]. Discussions involving sexual function should be a routine part of UMN or LMN cancer presentations. Patients should be educated about the underlying etiology of their sexual dysfunction in addition to available adaptive equipment, techniques and medications to achieve optimal sexual health. Involvement of psychology should also be considered in the management of sexual dysfunction of the cancer patient.

Chordoma patients present with a number of impairments that can be managed by physiatrists. For optimal bowel, bladder, and sexual management, identification of an UMN or LMN pattern is crucial. Ideally, a team approach for these patients between physiatrists, oncologists, gynecologists, gastroenterologists, urologists, surgeons, and therapists can improve functional independence and quality of life.

Acknowledgments We would like to thank Dr. Ross Zafonte, Dr. Kevin C. O'Connor, and Dr. Julie Silver for their assistance in the literature review and editorial contributions for this project. This work was supported by the Core Cancer Grant, P30 CA 008748.

\section{Compliance with ethical standards}

Conflict of interest The authors declare that they have no competing interests.

\section{References}

1. Boriani S, Bandiera S, Biagini R, Bacchini P, Coriani L, Cappuccio $\mathrm{M}$, et al. Chordoma of the mobile spine: fifty years of experience. Spine. 2006;31:493-503. 10.1097/1201.brs. 0000200038.30869.27.

2. McMaster ML, Goldstein AM, Bromley CM, Ishibe N, Parry DM, Chordoma: incidence and survival patterns in the United States, 1973-1995. Cancer Causes Control. 2001;12:1-11. 10.1023/ 123A:1008947301735.

3. Rich TA, Schiller A, Suit HD,Mankin HJ, Clinical and pathologic review of 48 cases of chordoma. Cancer. 1985;56:182-7. https:// doi.org/10.1002/1097-0142(19850701)56:1<182::aidcncr2820560131>3.0.co;2-j.

4. Walcott BP, Nahed BV, Mohyeldin A, Coumans JV, Kahle KT, Ferreira MJ. Chordoma: current concepts, management, and future directions. Lancet Oncol. 2012;13:e69-76. 10.1016/S1470-2045 (11)70337-0. 
5. Casali PG, Stacchiotti S, Sangalli C, Olmi P, Gronchi A. Chordoma. Curr Opin Oncol. 2007;19:367-70.

6. Azzarelli A, Quagliuolo V, Cerasoli S, Zucali R, Bignami P, Mazzaferro V, et al. Chordoma: natural history and treatment results in 33 cases. J Surg Oncol. 1988;37:185-91.

7. Samson IR, Springfield DS, Suit HD, Mankin HJ. Operative treatment of sacrococcygeal chordoma. J Bone Joint Surg Am. 1993;75-A:1476-84.

8. Healey JH, Lane JM. Chordoma: a critical review of diagnosis and treatment. Orthop Clin North Am. 1989;20:417-26.

9. Chandawarkar RY. Sacrococcygeal chordoma: review of 50 consecutive patients. World J Surg. 1996;20:717-9. $10.1007 / \mathrm{s} 002689900110$.

10. Smith J, Ludwig RL, Marcove RC. Skeletal radiology sacrococcygeal chordoma. Skeletal Radiol. 1987;16:37-44.

11. Osler P, Bredella MA, Hess KA, Janssen SJ, Park CJ, Chen YL, et al. Sacral insufficiency fractures are common after high-dose radiation for sacral chordomas treated with or without surgery. Clin Orthop Relat Res. 2015;474:1-3. 10.1007/s11999-015-46519.

12. Bergh P, Kindblom LG, Gunterberg B, Remotti F, Ryd W, Meis-Kindblom JM. Prognostic factors in chordoma of the sacrum and mobile spine: a study of 39 patients. Cancer. 2000;88:2122-34. 10.1002/(SICI)1097-0142(20000501) 88:9<2122::AID-CNCR19>3.0.CO;2-1.

13. Baratti D, Gronchi A, Pennacchioli E, Lozza L, Colecchia M, Fiore M, et al. Chordoma: natural history and results in 28 patients treated at a single institution. Ann Surg Oncol. 2003;10:291-6. 10. 1245/ASO.2003.06.002.

14. Cheng EY, Ozerdemoglu RA, Transfeldt EE, Thompson RC. Lumbosacral chordoma. Prognostic factors and treatment. Spine.1999;24:1639-45. 10.1097/00007632-199908150-00004.

15. Kayani B, Hanna SA, Sewell MD, Saifuddin A, Molloy S, Briggs TW. A review of the surgical management of sacral chordoma. Eur J Surg Oncol. 2014;40:1412-20. 10.1016/123j.ejso.2014.04.008.

16. Papagelopoulos PJ, Mavrogenis AF, Galani EC, Savvidou OD, Boscainos PJ, Katonis PG, et al. Chordoma of the spine: clinicopathological features, diagnosis, and treatment. Ortho Blue J. 2004;27:1256-63.
17. Fourney DR, Gokaslan ZL. Current management of sacral chordoma. Neurosurg Focus. 2003;15:E9. 10.3171/1foc.2003.15.2.9.

18. Stener B, Gunterberg B. High amputation of the sacrum for extirpation of tumors. Principles and technique. Spine. 1978;3:351-66.

19. Ji T, Guo W, Yang R, Tang X, Wang Y, Huang L. What are the conditional survival and functional outcomes after surgical treatment of 115 patients with sacral chordoma? Clin Orthop Relat Res. 2016. 10.1007/s11999-016-4773-8.

20. Schwab JH, Healey JH, Rose P, Casas-Ganem J, Boland PJ, The surgical management of sacral chordomas. Spine. 2009;34:2700-4. 10.1097/BRS.0b013e3181bad11d.

21. Todd LT, Yaszemski MJ, Currier BL, Fuchs B, Kim CW, Sim FH. Bowel and bladder function after major sacral resection. Clin Orthop Relat Res. 2002;397:36-9.

22. Hulen CA, Temple HT, Fox WP, Sama AA, Green BA, Eismont FJ, Oncologic and functional outcome following sacrectomy for sacral chordoma. J Bone Joint Surg Am. 2006;88:1532-9. 10. 2106/BJS.D.02533.

23. Wuisman P, Lieshout O, Sugihara S, van Dijk M. Total sacrectomy and reconstruction: oncologic and functional outcome. Clin Orthop Relat Res. 2000;381:192-203.

24. Gunterberg B, Kewenter J, Petersén I, Stener B. Anorectal function after major resections of the sacrum with bilateral or unilateral sacrifice of sacral nerves. Br J Surg. 1976;63:546-54.

25. Alexander MS, Biering-Sorensen F, Bodner D, Brackett NL, Cardenas D, Charlifue S, et al. International standards to document remaining autonomic function after spinal cord injury. Spinal Cord. 2009;47:36-43.

26. Sporer A, Leyson JF, Martin BF. Effects of bethanechol chloride on the external urethral sphincter in spinal cord injury patients. J Urol. 1978;120:62-6.

27. Finkbeiner A. Is bethanechol chloride clinically effective in promoting bladder emptying? A literature review. J Urol. 1985;134:443-9.

28. Ho CH, Sung KC, Lim SW, Liao CH, Liang FW, Wang JJ, et al. Chronic indwelling urinary catheter increase the risk of bladder cancer, even in patients without spinal cord injury. Medicine.2015;94:e1736. 10.1097/MD.0000000000001736. 